\title{
UNA NOTA SOBRE EL GOBIERNO CORPORATIVO EN LA INDUSTRIA BANCARIA
}

\author{
César Portalanza ${ }^{a}$ Guibell Carlevaro ${ }^{B}$
}

\begin{abstract}
RESUMEN
El presente documento pretende identificar determinadas características de la industria bancaria que hacen que el gobierno corporativo sea distinto al que se promueve para empresas que no pertenecen al sistema financiero. Las buenas prácticas de gobierno corporativo, desde la perspectiva de la $\mathrm{OECD}^{1}$, señalan un énfasis en la protección de los accionistas minoritarios de las empresas que cotizan en el mercado de valores; mientras que, por sus características particulares, entre ellas, el muy elevado apalancamiento, la regulación, y su gran importancia en la economía, el gobierno corporativo en la banca debe considerar que existen otros grupos de interés, además de los accionistas minoritarios, como los ahorristas, que requieren el resguardo de sus intereses, así como aspectos de gobierno orientados a la gestión de riesgos y control, y sistema de remuneraciones y compensaciones, como lo plantea el Comité de Basilea.
\end{abstract}

JEL: G30; G34; G38.

PALABRAS CLAVES: Gobierno Corporativo, Directorio, Gerencia

\begin{abstract}
This document aims to identify certain characteristics of the banking industry that make corporate governance different from that promoted for companies that do not belong to the financial system. Sound corporate governance practices, from the OECD perspective, point to an emphasis on the protection of minority shareholders of companies listed on the stock market; while, due to its particular characteristics, among them, the very high leverage, regulation, and its great importance in the economy, corporate governance in banking must consider that there are other interest groups, in addition to minority shareholders, such as depositors, who require the safeguarding of their interests, as well as aspects of government oriented to risk management and control, and remuneration and compensation system, as proposed by the Basel Committee.
\end{abstract}

JEL: G30; G34; G38.

KEYWORDS: Corporate Governance, Board of Directors, Management

\footnotetext{
A Investigador docente de la Escuela Profesional de Economía de la Facultad de Ciencias Contables, Económicas y Financieras de la Universidad de San Martín de Porres.

B Licenciada en Administración de la Universidad Nacional Mayor de San Marcos.

1 OECD (2015). G20/OECD Principles of Corporate Governance. OECD Publishing, Paris.
} 


\section{INTRODUCCIÓN}

Los últimos desastres financieros, fraudes, malversación de fondos, falsificación contable entre otros, ocurridos a nivel mundial, han manifestado la desconfianza de los clientes en las empresas y, como consecuencia, su imagen ha sido deteriorada. La implementación de buenas prácticas de gobierno corporativo puede lograr un balance entre el riesgo y la rentabilidad en un horizonte de sostenibilidad de mediano y largo plazo con el objeto de intentar evitar desastres financieros empresariales.

Laimportancia de las buenas prácticas de gobierno corporativo se ha destacado recientemente a partir de los desastres financieros, que han puesto en duda lo que usualmente se asume que existe en las empresas, el uso de buenas prácticas para la toma de decisiones empresariales; en particular, se destacan, entre otras, la confusión de roles, responsabilidades y funciones del directorio y la gerencia.

\section{PROBLEMA DEL PRINCIPAL-AGENTE Y GOBIERNO CORPORATIVO}

Las empresas pueden realizar sus actividades empresariales debido a que logran financiar sus negocios, típicamente a través de capital y deuda, ambas en sus diversas modalidades. Con este aporte de capital, los accionistas obtienen derechos políticos y económicos, entre ellos elegir al directorio, que de acuerdo a la OECD (2015): “...es responsable de controlar los resultados de la dirección y ofrecer una rentabilidad adecuada a los accionistas, al tiempo que debe evitar conflictos de interés y lograr un equilibrio entre las exigencias contrapuestas que afronta la empresa"'2.

En este contexto empresarial, los accionistas eligen al directorio. A su vez, el directorio, para la gestión de la empresa, elige a la gerencia. En esta secuencia de designaciones, la pregunta que surge es si el directorio actuará de acuerdo a los intereses de quienes los eligen, los accionistas; en el mismo sentido, también nos podemos preguntar, si la gerencia actuará de acuerdo a los lineamientos dados por el directorio. En teoría, cada grupo de interés, en este caso directorio y gerencia, deberían alinearse a los intereses de quienes los eligen; sin embargo, el gerente puede contratar más personal del necesario o puede estar muy enfocado en sus intereses particulares y no en la generación de valor para el accionista. Este es el problema que se conoce como el del

2 OCDE,p. 51.(Ver referencias)
Principal - Agente de la Teoría de Agencia. Jensen y Meckling (1976) señalan:

We define an agency relationship as a contract under which one or more persons (the principal(s)) engage another person (the agent) to perform some service on their behalf which involves delegating some decision making authority to the agent. If both parties to the relationship are utility maximizers, there is good reason to believe that the agent will not always act in the best interests of the principal. ${ }^{3}$

Más enfocado en los accionistas, Shleifer y Vishny (1997) concluyen, “Corporate governance deals with the agency problem: the separation of management and finance. The fundamental question of corporate governance is how to assure financiers that they get a return on their financial investment" 4 .

Por lo que, el buen gobierno corporativo plantea soluciones al problema de agencia, entendiendo que los proveedores de capital en la empresa son los accionistas, mayoritarios o minoritarios, $\mathrm{y}$ estos últimos sin mayor control sobre el management. Como señala Tirole (1999), "La definición común de gobierno corporativo promueve la defensa de los intereses de los inversionistas (accionistas) ${ }^{\prime \prime}$.

En este sentido, las recomendaciones de la OCDE, en su primera y segunda revisión en el 2004 y 2015 , respectivamente, entre otras recomendaciones, están orientadas a promover la protección de los accionistas minoritarios de las empresas que cotizan en el mercado de capitales, sean estas instituciones financieras o no.

Las decisiones del management afectan a todos los grupos de interés, de dentro y fuera de la empresa, que contribuyen con el financiamiento y desarrollo de la empresa (acreedores, proveedores, clientes, entre otros). Es así, que a los que actúan financiando la empresa, deuda y equity, les interesa que el negocio sea viable, aunque no todos los grupos de interés participan en la elección del directorio (sólo lo hacen los accionistas).

Tirole (1999) señala que las decisiones del management generan externalidades a diversos

\footnotetext{
3 Jensen, M. C and Meckling, W. H., p. 5.(Ver referencias)

4 Shleifer, A. and R. Vishny., p.773.(Ver referencias)

5 Tirole, J., p.9.(Ver referencias)

6 OECD (2015). G20/OECD Principles of Corporate Governance. OECD Publishing, Paris.
} 
grupos de interés ("natural stakeholders"), además de los accionistas minoritarios, a los acreedores, proveedores, clientes, entre otros. La implementación de buenas prácticas de gobierno corporativo tiene como objetivo garantizar un sistema de "pesos y contrapesos" que generen beneficios a todos los grupos de interés, lo que permite reducir los conflictos de agencia.

\section{EL GOBIERNO CORPORATIVO EN LA INDUSTRIA BANCARIA}

La industria bancaria ${ }^{7}$ tiene características que la hacen muy diferente a otros sectores o actividades económicas. Entre otras características, quisiéramos destacar su importancia en la economía, la regulación, y el elevado nivel de apalancamiento.

El gobierno corporativo en la industria bancaria debe tener claramente elementos diferenciadores de empresas que desarrollan actividades no bancarias. Como señala Hagendorff $(2015)^{8}$, el gobierno corporativo tiene elementos comunes entre la banca y otras industrias; sin embargo, sí existen determinadas características que las diferencian como el sistema de compensaciones y remuneraciones, el directorio, la estructura de capital, y la gestión de riesgos.

Adam y Mehran (2003) señalan que, los accionistas, ahorristas y reguladores hacen que sea más complejo el gobierno de los bancos:

The governance of banking firms may be different from that of unregulated, nonfinancial firms for several reasons. For one, the number of parties with a stake in an institution's activity complicates the governance of financial institutions. In addition to investors, depositors and regulators have a direct interest in bank performance. On a more aggregate level, regulators are concerned with the effect governance has on the performance of financial institutions because the health of the overall economy depends upon their performance. ${ }^{9}$

\section{Importancia de la industria bancaria en la economia}

La industria bancaria es trascendental en el progreso de la economía, debido a que en su rol

\footnotetext{
7 Entiéndase por "bancos" a cualquier institución del sistema financiero (bancos, cajas, etc.) que cumplen en rol de intermediarios financieros en la economía.

8 Hagendorff, J., p.1. (Ver referencias)

9 Adams, R., and H. Mehran, p. 124. (Ver referencias)
}

de intermediario financiero canaliza el ahorro de la economía hacia el desarrollo de actividades productivas $^{10}$, promoviendo el financiamiento empresarial y el desarrollo de inversiones. El buen funcionamiento de la banca favorece el crecimiento económico, por lo que un sistema financiero solvente, estable y eficiente dinamiza el crecimiento de la economía de un país.

\section{Industria regulada}

Una característica fundamental del sistema bancario es que se encuentra sujeto a una regulación, que comprende, entre otros, requerimientos mínimos de capital, límites operativos, y determinados requerimientos de buen gobierno. Hay que señalar que cada decisión importante dentro de la organización está altamente influenciada por el gobierno interno (Directorio) y por el gobierno externo, principalmente el regulador bancario, y el mercado. No todas estas fuerzas son iguales y sus intereses no siempre se alinean, ya que no siempre buscan el mismo resultado ${ }^{11}$. En esta industria existe una alta regulación pero la gestión de los activos bancarios sigue siendo responsabilidad del banco.

La regulación macroprudencial tiene como objetivo garantizar la estabilidad del sistema financiero, mientras que, la regulación microprudencial está enfocada en proteger a los ahorristas, además para dicho fin, existe el fondo de seguro de depósitos, que amortigua las pérdidas de los ahorristas.

\section{Elevado apalancamiento bancario}

El apalancamiento es otro elemento central en el negocio bancario. El financiamiento del negocio bancario proviene de recursos de terceros (depósitos), y el equity representa sólo un diez por ciento del financiamiento total. En el Cuadro 1, se presenta un balance muy simplificado con el objetivo de destacar el alto grado de apalancamiento en esta industria, y se puede apreciar que, los accionistas aportan 1 y gestionan un negocio de $14.5^{12}$, y son estos accionistas los que eligen a un directorio que resguarde sus intereses.

\footnotetext{
10 Allen, F., Carletti, E. and Gu, X., p.1. (Ver referencias)

11 Mehran, H. y Mollineaux, L., p 11. (Ver referencias)

12 Se supone que los activos y contingentes ponderados por riesgo (AyCPR) equivalen a 10. En este aspecto, debemos señalar que, Basilea III (diciembre 2017) está recomendando limitar las operaciones de los bancos no sólo con el ratio de capital típico o ratio Cooke sino con un ratio apalancamiento, limitando la relación de los activos y contingentes sin ponderar respecto del capital regulatorio.
} 
En esta estructura de capital (9 de Depósitos y 1 de equity), hay un incentivo natural hacia una mayor toma de riesgos en los proyectos o inversiones por parte del banco debido a que dichos proyectos son financiados con recursos de terceros, no con dinero del accionista. Si un proyecto genera un valor presente neto (VPN) positivo, beneficia a los accionistas; mientras que, si el VPN es negativo las pérdidas son asumidas por los accionistas pero fundamentalmente por quienes han financiado en gran parte el proyecto, como son los ahorristas.

\section{Cuadro 1. Balance bancario simplificado y estructura del activo y contingente del total de la banca múltiple a diciembre 2018}

\begin{tabular}{|l|r|l|r|}
\hline Disponible & 1.50 & Depósitos & 9.00 \\
Inversiones & 1.20 & Equity & 1.00 \\
Créditos & 7.00 & & \\
Otros & 0.30 & & \\
\hline \multirow{2}{*}{ Total Activos } & $\mathbf{1 0 . 0 0}$ & Total Depósitos y & Equity \\
\cline { 1 - 2 } Contingentes & $\mathbf{4 . 5 0}$ & & \\
\cline { 1 - 2 } & $\mathbf{1 4 . 5 0}$ & & \\
\cline { 1 - 2 } & &
\end{tabular}

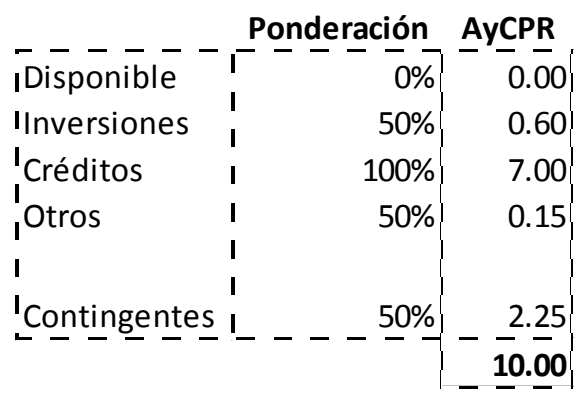

Ratio de Capital

\section{Balance Bancario Simplificado}

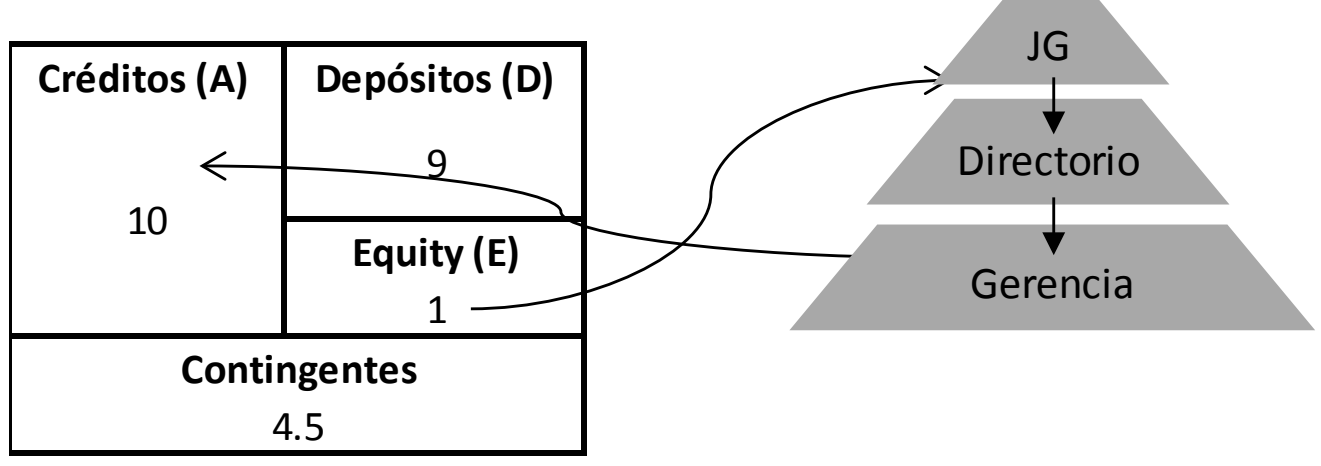

Elaboración propia.

\section{Gobierno corporativo en la OCDE y Basilea}

Esta particularidad de la industria bancaria es la que le permite al Comité de Basilea sobre Supervisión Bancaria identificar elementos diferenciadores en sus "Principios sobre Gobierno Corporativo para la Banca"13. El Comité de Basilea destaca, entre otros, la separación de funciones y responsabilidades del Directorio y la Gerencia, la Gestión de Riesgos,

13 Bank for International Settlements. (2015). (Ver referencias) el Control Interno y Cumplimiento, y el Sistema de Compensaciones.

Respecto del directorio, se establece que es el máximo responsable de la dirección del banco, y tiene, entre otras funciones, la supervisión de la Gerencia, y ésta tiene la función de reportar al Directorio. Por otro lado, la gestión de riesgos es otra característica intrínseca en el negocio bancario, que requiere de buenas prácticas en el gobierno para evitar la toma excesiva de riesgos en el corto plazo que afecte la sostenibilidad del 
banco. Los Principios de Gobierno Corporativo de Basilea también recomiendan un sistema de remuneraciones y compensaciones que penalice la toma de riesgos desalineados con los establecidos por el Directorio, con el fin de eliminar incentivos perversos que afecten el patrimonio.

\section{Cuadro 2. Principios de Gobierno Corporativo según la $\mathrm{OCDE}^{14}$ y el Comité de Basilea $^{15}$}

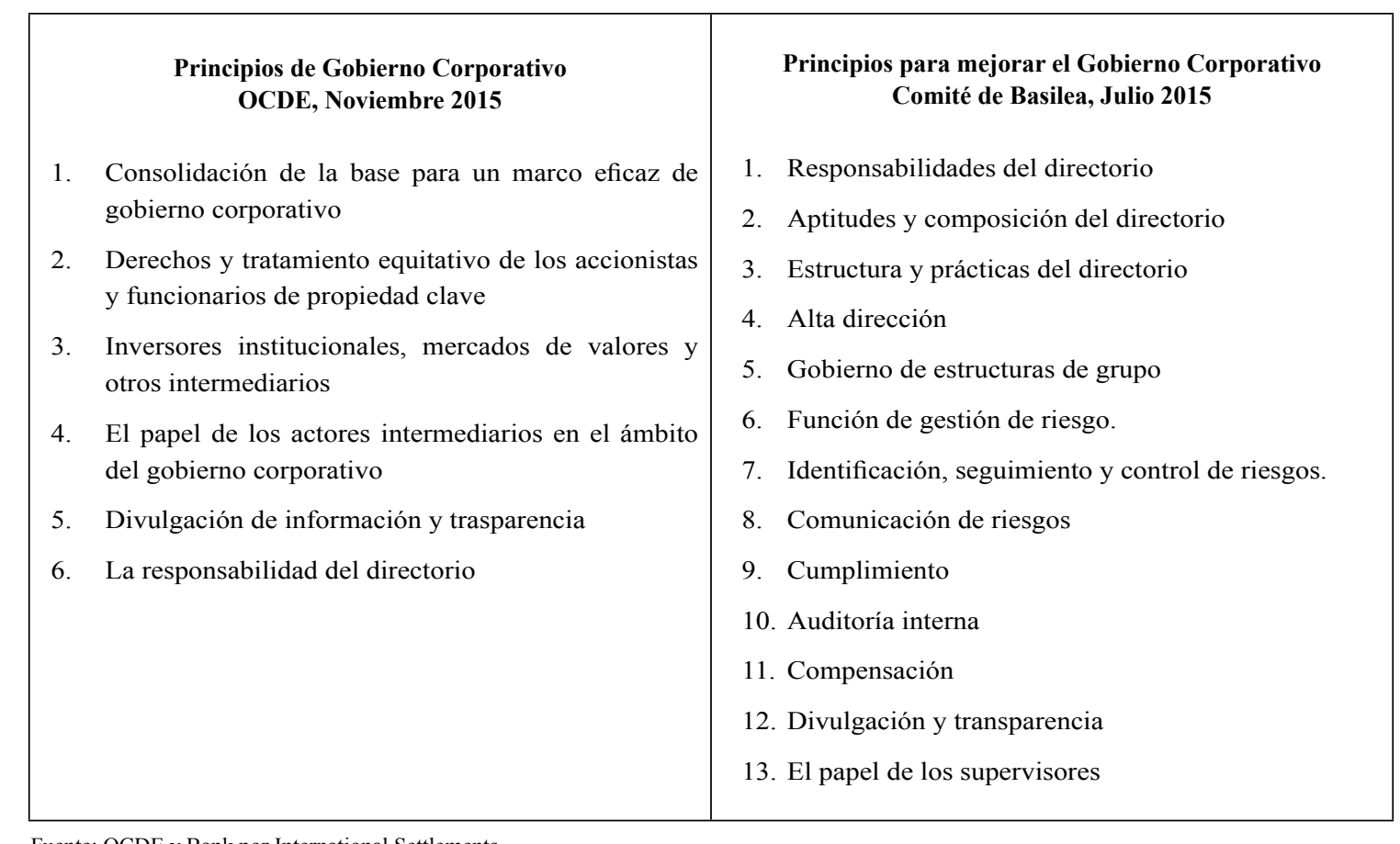

Fuente: OCDE y Bank por International Settlements.

En el Cuadro 1 se aprecia que los accionistas aportan 1, y dirigen un negocio de 14.5; además, estos accionistas eligen al Directorio con el objeto de que proteja sus intereses. En el balance simplificado se evidencia que los depositantes, a pesar de que financian el negocio bancario en un $90 \%$ no tienen una injerencia en la administración, por lo que resulta razonable que desde el Comité de Basilea se promueva la participación de independientes en el directorio (Principio 2), que por su imparcialidad garanticen un tratamiento objetivo a este grupo de interés, que es el que financia el negocio bancario.

Debe mencionarse que, la regulación bancaria si bien protege los intereses de los ahorristas, no tiene un rol directo ni activo en la gestión del banco como sí lo tiene el Directorio (en representación de los accionistas) y la Gerencia. El Comité de Basilea promueve la participación de directores independientes para velar por los intereses de los ahorristas y de todos los grupos de interés; no obstante, los "modelos" de gobierno corporativo en el mundo difieren en el tratamiento del directorio, como lo señala Haggendorff (2015), en Alemania los trabajadores tienen participación en los directorios de las grandes empresas ${ }^{16}$.

Finalmente, como Haggendorff (2015), debemos señalar que las buenas prácticas de gobierno corporativo en la banca deben estar vinculadas a las propias características del negocio bancario, y entre ellas más focalizadas a velar por los intereses de quienes financian este negocio, como se observa en el Cuadro 1. 
The corporate governance of banks is clearly an important topic both for investors and for policymakers. However, much of what is known about the governance of banks has relied on replicating research based on the nonfinancial sector without adequately taking the unique features of banks into account. However, these unique features of banks call for a more profound rethink of the corporate governance of banks, one that centers around debtholders rather than equity holders....

\section{CONCLUSIONES}

Una característica central en el negocio bancario es su muy elevado nivel de apalancamiento; sin embargo, los acreedores no tienen mayor injerencia en la administración del banco, pero sí pueden resultar perjudicados por el management y los accionistas. Una recomendación del Comité de Basilea (2015) es la de tener directores independientes, con el objetivo de garantizar que los beneficios y perjuicios se distribuyan objetivamente entre los diferentes grupos de interés.

El Comité de Basilea (2015), debido a las características propias del negocio bancario, plantea recomendaciones sobre la gestión de riesgos, control y cumplimiento, así como un sistema de remuneraciones e incentivos que aliente la sostenibilidad a mediano y largo plazo del banco. Los Principios de Gobierno Corporativo de la OCDE no consideran en sus recomendaciones los temas planteados por el Comité de Basilea.

\section{REFERENCIAS}

[1] Adams, R., and H. Mehran. (2003). Is Corporate Governance Different for Bank Holding Companies? Economic Policy Review 9(1):123-42.

[2] Allen, F., Carletti, E., and Gu, X. (2008). The Roles of Banks in Financial Systems. In The Oxford Handbook of Banking, Edited by Allen N. Berger, Phillip Molyneux, and John O.S. Wilson.

[3] Bank for International Settlements (2015). Corporate Governance principles for Banks.

[4] Claessens, S. y B. Yurtoglu. (2012). Corporate Governance and Development: An Update. Focus $\mathrm{N}^{\circ}$ 10. Global Corporate Governance Forum and IFC. Washington DC.
[5] De Haan, Jakob, y Vlahu, Razvan. (2013). Corporate Governance of Banks: A survey. DNB Working Paper. Amsterdam.

[6] Harm, Christian (2002). Bank Management between Shareholders and Regulators. Société Universitaire Européenne de Recherches Financiéres.

[7] Hagendorff, Jens (2015). Corporate Governance in Banking. In The Oxford Handbook of Banking, Edited by Allen N. Berger, Phillip Molyneux, and John O.S. Wilson. Second Edition.

[8] Jensen, M y Meckling, W. (1976). Theory of the firm: Managerial behavior, agency costs and ownership structure. Journal of Financial Economics.

[9] Kenneth J. Arrow. (1986). "Agency and the Market”. In Handbook of Mathematical Economics, vol III, ed. Kenneth J Arrow and Michael D Intriligator (Amsterdam: Elsevier Science Publishers B.V. North-Holland).

[10] Maher, Maria y Andersson, Thomas. (1999). Corporate Governance: Effects on Firm Performance and Economic Growth. Organization for Economic Co-Operation and Development (OECD).

[11] Mehran, Hamid y Mollineaux, Lindsay. (2012). Corporate Governance of Financial Institutions. Federal Reserve Bank of New York Staff Report. No. 539.

[12] Mehran, H., Morrison, A., y Shapiro, Joel. (2011). Corporate Governance and Banks: What Have We Learned from the Financial Crisis? Federal Reserve Bank of New York Staff Reports. No. 502.

[13] Mehran, H., y L. Mollineaux (2012). Corporate Governance of Financial Institution. Federal Reserve Bank of New York. Staff Reports N 539. February 2012.

[14] Minton, B., Taillard, J., y Rohan Williamson. (2014). Financial Expertise of the Board, Risk Taking, and Performance: Evidence from Bank Holding Companies. Journal of Financial and Quantitative Analysis. Vol. 49, No. 2. pp. 351-380. 
[15] Mülbert, Peter. 2010. Corporate Governance of Banks after the Financial Crisis - Theory, Evidence, Reforms. ECGI Working Paper $\mathrm{N}^{\circ} 151 / 2010$.

[16] OCDE. (2015). Principios de Gobierno Corporativo de la OCDE y del G20. Editions OCDE, Paris. http://dx.doi. org/10.1787/9789264259171-es.

[17] Shleifer A, Vishny R. (1986).Large Shareholders and Corporate Control, Journal of Political Economy, 94(3): 46188.

[18] Stenning, Francis, Boza, Beatriz (2015). Buenas prácticas de Gobierno Corporativo. La Voz del Mercado.
[19] Stulz, René Z. (2016). Risk Management, Governance, Culture, and Risk Taking in Banks. En FRBNY Economic Policy Review.

[20] The Committee on the Financial Aspects of Corporate Governance. The Cadbury Report, December 1992, http://cadbury. cjbs.archios.info/report.

[21] Tirole, J. (1999). El Gobierno Corporativo. En Economía Vol. XXII, N 44. Diciembre. PUCP. 